\title{
openheart Prehospital identification of ST-segment elevation myocardial infarction and mortality (ANZACS-QI 61)
}

\author{
Becky Yi-Wen Liao (D) , ${ }^{1,2}$ Mildred Ai Wei Lee, ${ }^{1}$ Bridget Dicker (D) ,3,4 \\ Verity F Todd, ${ }^{3,4}$ Ralph Stewart (D) , ${ }^{2}$ Katrina Poppe (D) , ${ }^{5}$ Andrew Kerr ${ }^{5,6}$
}

\begin{abstract}
- Additional supplemental material is published online only. To view, please visit the journal online (http://dx.doi.org/10. 1136/openhrt-2021-001868).

To cite: Liao BY-W, Lee MAW, Dicker B, et al. Prehospital identification of ST-segment elevation myocardial infarction and mortality (ANZACS-QI 61). Open Heart 2022;9:e001868. doi:10.1136/

openhrt-2021-001868
\end{abstract}

Received 22 September 2021 Accepted 5 January 2022

Check for updates

(C) Author(s) (or their employer(s)) 2022. Re-use permitted under CC BY-NC. No commercial re-use. See rights and permissions. Published by BMJ.

For numbered affiliations see end of article.

Correspondence to Dr Becky Yi-Wen Liao; beckywliao@gmail.com

\section{ABSTRACT}

Background Early recognition of ST-segment elevation myocardial infarction (STEMI) is needed for timely cardiac monitoring and reperfusion therapy.

Methods Three anonymously linked New Zealand national datasets (July 2016-November 2018) were used to assess the utilisation of ambulance transport in STEMI cases, the concordance between ambulance initial clinical impressions and hospital STEMI diagnoses, and the association between initial paramedic clinical impressions and 30-day mortality. The St John Ambulance electronic record captures community call-outs and paramedic initial clinical impressions. The national cardiac (ANZACS-QI) registry and national administrative datasets capture all New Zealand public hospital admission diagnoses and mortality data.

Results 0 f 5465 patients with STEMI, $73 \%$ were transported to hospital by ambulance. For these patients, the initial paramedic impression was STEMI in 50.7\%, another acute coronary syndrome (ACS) diagnosis in $19.9 \%$ and non-ACS diagnosis in $29.7 \%$. Only $37 \%$ of the 5465 patients with STEMI were both transported by ambulance and clinically suspected of STEMI by paramedics. Compared with patients with paramedic'suspected STEMl', 30-day mortality was over threefold higher for patients thought to have a non-ACS condition (10.9\% and $34.9 \%$, respectively), but after adjustment for available covariates, this was substantially ameliorated (HR 1.48, 95\% Cl 1.22 to 1.80).

Conclusions In this national data linkage study, only 4 out of every 10 patients with STEMI were both transported by ambulance and had STEMI suspected by paramedics. Although patients with STEMI not suspected of an ACS diagnosis by paramedics had the highest mortality rate, this is largely explained by the different risk profile of these patients.

\section{INTRODUCTION}

Timely and accurate diagnosis of ST-segment elevation myocardial infarction (STEMI) is a prerequisite for implementation of optimal evidence-based treatments including antithrombotic therapy and coronary reperfusion. ${ }^{1-8}$ The St John Ambulance service is a charity with funding from government and community donations, attends to most

\section{Key questions}

What is already known about this subject?

- Timely and accurate diagnosis of ST-segment elevation myocardial infarction (STEMI) is important to facilitate cardiac monitoring and acute reperfusion therapy. Prior studies have reported variable accuracy in STEMI diagnoses by paramedics, but the overall proportion of all hospitalised patients with STEM who are transported by ambulance and had STEM suspected by paramedics is not known.

What does this study add?

- This national data linkage study anonymously linked the New Zealand national cardiac (ANZACS-QI) registry, national administrative datasets and the St John Ambulance electronic records to identify all patients hospitalised with STEMI. This allowed us to report the proportion presenting via ambulance and the accuracy of the paramedic clinical impressions. Only 4 out of every 10 hospitalised patients with STEMI were both transported by ambulance and had STEMI suspected by paramedics. Patients with STEMI who were not suspected of an acute coronary syndrome diagnosis by paramedics had a higher mortality rate than those suspected of STEMI or who self-transported to hospital, although this is largely explained by the different risk profile of these patients.

How might this impact on clinical practice?

- Increasing both the proportion of patients transferred by ambulance and improving identification of at-risk patients by paramedics are needed to optimise the known benefits of early and accurate STEMI identification.

of the medical emergency call-outs in New Zealand (NZ). Virtually all emergency hospitalisations in NZ are admitted to public hospitals, including over $99 \%$ of acute coronary syndrome (ACS) admissions. ${ }^{9}$ In 2016, St John Ambulance implemented an electronic clinical record which contains information on patient clinical status and the paramedic initial clinical impression, including 'suspected STEMI' and 'other suspected 
cardiac'. Anonymised linkage of individual ambulance electronic records with public hospitalisation and mortality databases allows us to construct a comprehensive dataset of ambulance-to-hospital transfers, hospitalisations and outcomes for most of NZ. These linked datasets were used to investigate the utilisation of ambulance transport in STEMI cases, and to evaluate the concordance between the paramedic initial clinical impressions and the final hospital diagnoses. Our aims were to determine the proportion of patients with a final hospital diagnosis of STEMI who were transported by ambulance with a paramedic-'suspected STEMI', and to assess the impact of discordance between prehospital and final STEMI diagnoses on mortality outcomes.

\section{METHOD}

This national data linkage study included consecutive cases captured in each of the three health datasets between 01 July 2016 and 30 November 2018.

\section{Terms used in this study}

The definition of 'suspected STEMI' in St John records required both clinical impression and ECG suggestive of STEMI by paramedics. When their initial impression is ACS or other cardiac conditions but felt not to meet the criteria for STEMI, then it is classified as 'other suspected ACS'. The initial clinical impression is generally that of the paramedic prior to any electronic transmission of the ECG to an interventional centre. Remaining cases which do not meet either definition are classified as 'ACS not suspected'.

\section{Datasets}

The St John electronic record system collects information for all ambulance call-outs, including patient demographics, clinical indications, transport times, and prehospital assessment and management. The ambulance data were obtained for the whole of NZ except for patients transported to Wellington, Hutt and Wairarapa Hospitals because they use a different ambulance service (contributing only $10 \%$ of the NZ total).

The All NZ Acute Coronary Syndrome Quality Improvement (ANZACS-QI) registry has captured over 99\% of NZ patients with ACS investigated with coronary angiography since 2015. The dataset and methodology of the ANZACS-QI registry have been previously reported, capturing ACS diagnosis types-STEMI, non-ST-elevated ACS (NSTEACS) and non-ACS diagnoses. ${ }^{10}$

The national administrative datasets include the National Minimum Dataset (NMDS) which provides International Classification of Diseases (ICD) 10AM-coded hospitalisation records, the Mortality Collection that provides mortality data, and the National Non-Admitted Patient Collection (NNAPC) which records patients presented to the emergency department who do not require hospitalisation and are discharged alive. NNAPC does not record ICD-coded diagnostic information. Online supplemental table 1 shows the ICD-10AM codes used to define ACS diagnosis subtypes in the NMDS (STEMI, non-STEMI (NSTEMI), unstable angina (USA), myocardial infarction (MI) unspecified), and other coronary heart disease diagnoses. Both primary and secondary codes were used. Age, gender, ethnicity and an area-based index of socioeconomic deprivation (New Zealand Deprivation Index 2013 (NZDep13)) ${ }^{11}$ were obtained from the national datasets. Ethnicity was prioritised using a modified version of NZ Standard Ethnicity Protocol. ${ }^{1211}$ Admission hospitals were classified as those participating in a routine primary percutaneous coronary intervention (PCI) service, those with a catheter laboratory but without routine primary PCI availability, or rural hospitals without a catheter laboratory. For STEMI cases, 'system time' was from ambulance dispatch to hospital arrival.

\section{Dataset linkage and cohort construction}

Every patient in contact with NZ health system is assigned a unique identifier, the National Health Index (NHI). The NHI can be used to anonymously link multiple electronic databases using a common encrypted NHI. ${ }^{10}$

Combined National Dataset: ACS hospitalisation episodes in the NMDS were defined using a previously validated 'bundling' method to account for interhospital transfers within a single ACS episode of care. ${ }^{9}$ For NMDS ACS bundles with more than one ACS diagnosis, ACS type was prioritised in the order STEMI $>$ NSTEMI $>\mathrm{USA}>\mathrm{MI}$ unspecified. When discrepancies arose between the final diagnoses in NMDS and ANZACS-QI, the latter was prioritised. The ANZACS-QI and NMDS admissions were linked and patients admitted to hospitals not in the St John catchment were excluded to form a Combined National Dataset.

St John dataset: from the raw St John dataset (789 466 records), duplicate entries and multiple call-outs of the same episode were excluded $(n=25865)$. A further 183428 records were excluded as they were records of hospital transfers, prehospital deaths or for patients not transferred to hospital, where hospitalisation diagnostic data were not available. The final 580173 St John acute hospital transports and 2256567 Combined National Dataset admissions were merged, resulting in a final cohort of 2396391 records for analysis (online supplemental figure 1).

\section{Outcomes}

The primary endpoint, 30-day post-admission all-cause mortality, was obtained for patients with a final STEMI diagnosis using the National Mortality dataset. These are reported for the first STEMI presentation per patient.

\section{Statistics}

Results were presented as mean with SD and/or median with IQR for continuous variables and frequency (percentage) for categorical variables. Comparison between groups (St John initial diagnosis or St John attendance) was done using Pearson's $X^{2}$ test for 
categorical data or non-parametric Mann-Whitney U test for continuous variables as the data were not normally distributed. The significance of the difference between the final diagnosis of STEMI according to the initial St John clinical impression and method of transport to hospital was tested using log-rank test. Unadjusted Kaplan-Meier curves showed the association between final diagnosis of STEMI according to initial St John clinical impression and method of transport to hospital, and mortality. The proportional hazard assumption was tested using SAS ASSESS statement in PROC PHREG and was met. Multivariable Cox regression models were used to estimate the adjusted hazard of ambulance paramedic 'other suspected ACS', 'ACS not suspected' and 'not attended by ambulance' compared with 'suspected STEMI' for 30-day mortality. Covariates included were age (continuous), gender, ethnicity, NZDep13, modified non-cardiac Charlson Comorbidity Index, coronary intervention status and hospital. All $\mathrm{p}$ values were two tailed and $\mathrm{p}<0.05$ was considered statistically significant. Data were analysed using SAS V.9.4 (SAS Institute), and survival plots were created using RStudio V.1.1.442.

\section{Patient and public involvement}

It was not appropriate to involve patients or the public in the design, conduct, reporting or dissemination plans of our research.

\section{RESULTS}

There were 5465 admissions with a final diagnosis of STEMI (table 1) and $3989(73 \%)$ were transported to hospital by ambulance and 1486 (27\%) self-presented. Of those transported by ambulance, $2016(50.7 \%)$ had a prehospital clinical impression of suspected STEMI, 792 (19.9\%) were suspected of another ACS diagnosis and 1181 (29.7\%) were not suspected of an ACS.

For the 3557 cases where paramedics suspected a STEMI, the positive predictive value was $57 \%$. Of the remaining cases, $16 \%$ were admitted with another coronary heart disease (CHD) diagnosis, predominantly NSTEACS. Another 21\% were admitted with non-cardiac diagnosis and $7 \%$ were alive but did not require hospital admission. Therefore, of all the cases suspected by paramedics of having STEMI, $72 \%$ had a final cardiac diagnosis.

Table 2 shows the characteristics of those cases with a final diagnosis of STEMI according to the initial paramedic impression and the mode of presentation. Cases identified as suspected STEMI by paramedics, as opposed to another diagnosis, were more likely to be younger and male. They also had less medical comorbidity when compared with those with a non-cardiac initial impression. Māori (45\%) or Pacific people (42\%) with STEMI were less likely to be initially identified as having a STEMI than Indian (57\%), other Asian (58\%) and European/ other $(52 \%)$ people. Only $36 \%$ of STEMI cases transported initially to rural hospitals without catheter laboratories were suspected STEMIs prehospital compared with $53 \%$ transported to hospitals participating in a routine primary PCI service and $47 \%$ to hospitals with a catheter laboratory but without routine primary PCI. The mean time from first medical contact to hospital arrival was shorter when St John suspected a cardiac condition (54.6, 54.0 and $59.3 \mathrm{~min}$ for suspected STEMI, other ACS and non-ACS, respectively $(\mathrm{p}<0.001))$. Self-presentation to hospital, in contrast to ambulance transfer, was more common among older patients, men and non-European/ other ethnic groups (Māori (36\%), Pacific (31\%), European/other $(24 \%))$.

Of the 3989 patients transported by ambulance with a final diagnosis STEMI, $2016(51 \%)$ had an initial paramedic clinical impression of STEMI. As a consequence of this diagnostic sensitivity, combined with $73 \%$ of patients being transported by ambulance, only $37 \%$ of all STEMI cases were identified prehospital as a suspected STEMI (table 3). This proportion varied by age-younger patients, men and European/other patients with STEMI were most likely to be transported by ambulance with a clinically suspected STEMI (European/other 38.7\% vs Māori and Pacific groups, $28.7 \%$ and $28.9 \%$ ) as were those without multiple comorbidities.

In the 38332 cases with an initial ambulance paramedic clinical impression of 'other suspected ACS', 792 (2.1\%)

Table 1 Ambulance initial clinical impressions and the final diagnosis available in the Combined National Dataset for all cases Final diagnosis in the Combined National Dataset (column \%)

\begin{tabular}{|c|c|c|c|c|c|c|}
\hline & STEMI & NSTEACS & Other CHD & Non-CHD & $\begin{array}{l}\text { Not } \\
\text { admitted }\end{array}$ & Total \\
\hline Total & 5465 & 28304 & 14862 & 2184334 & 163426 & 2396391 \\
\hline Suspected STEMI & $2016(36.9)$ & $472(1.7)$ & $86(0.6)$ & $733(0.1)$ & $250(0.2)$ & $3557(0.1)$ \\
\hline Other suspected ACS & $792(14.5)$ & $6876(24.3)$ & $2484(16.7)$ & $21931(1.0)$ & $6249(3.8)$ & $38332(1.6)$ \\
\hline
\end{tabular}

CHD, coronary heart disease; NSTEACS, non-ST-elevated acute coronary syndrome; STEMI, ST-segment elevation myocardial infarction. 


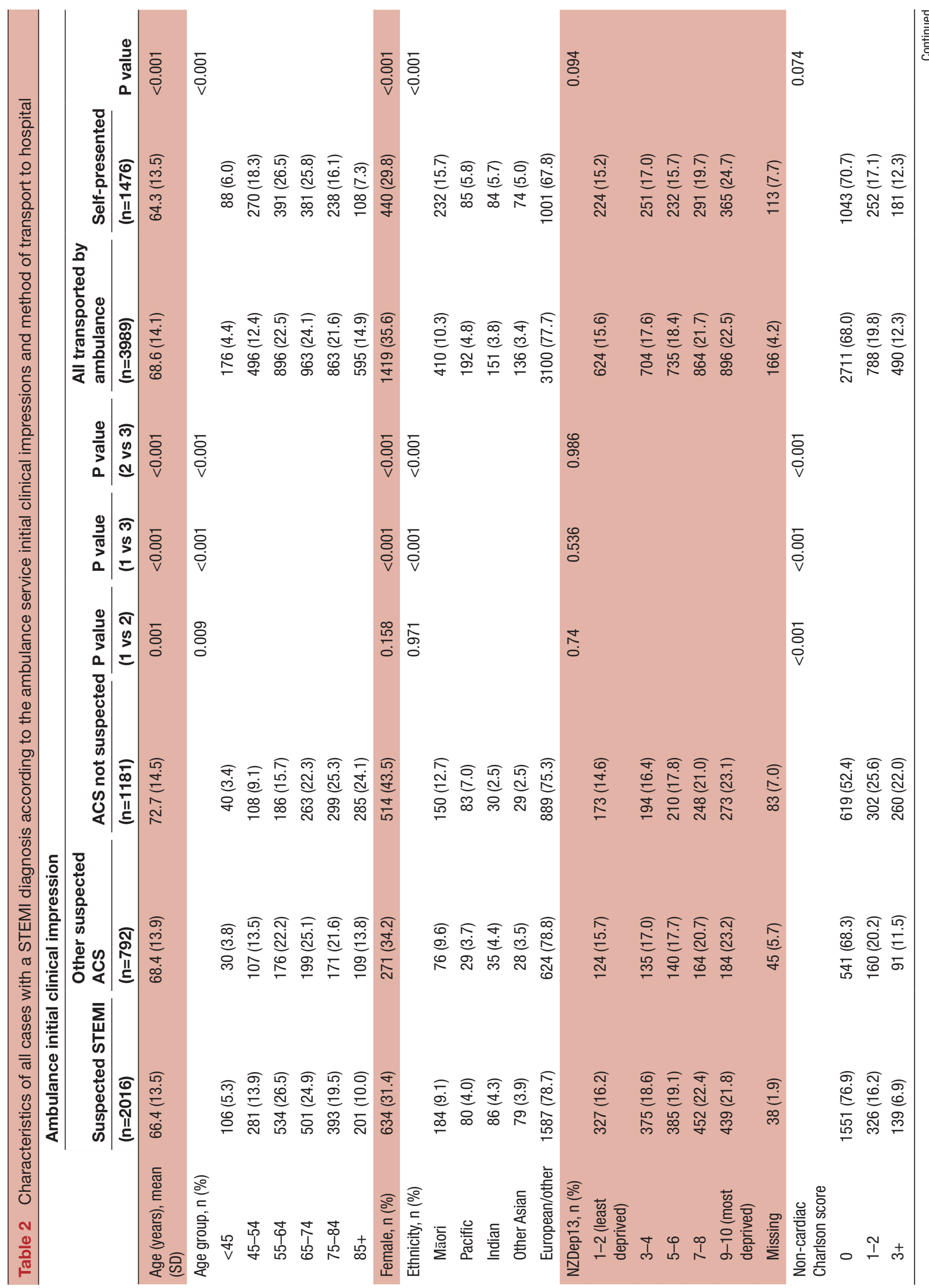




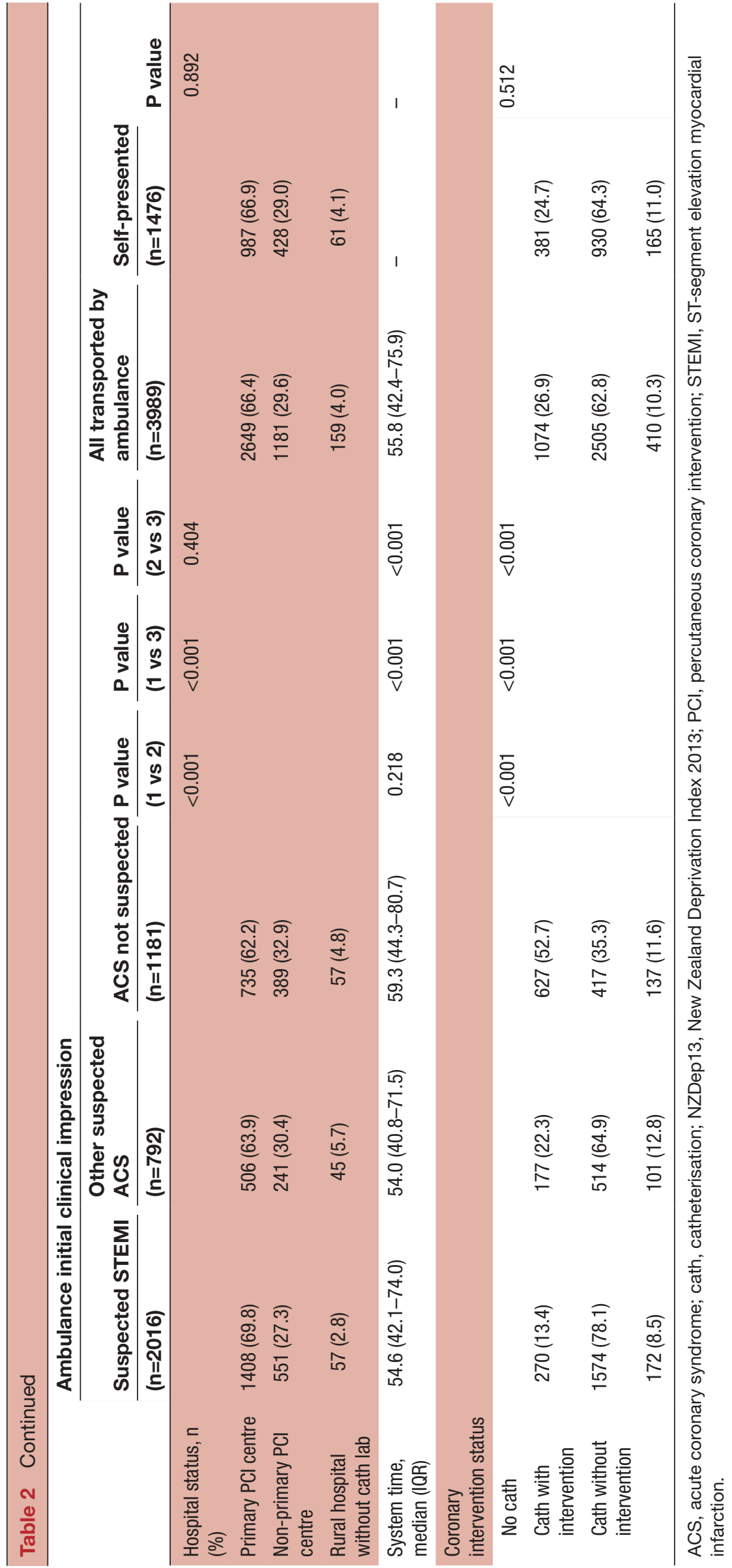


Table 3 Proportion of patients with a final diagnosis of STEMI who were transported by ambulance with a paramedic-suspected STEMI

Final STEMIs transported by ambulance and suspected of STEM prehospital/all final STEMI

\begin{tabular}{|c|c|}
\hline All & $2016 / 5465(36.9 \%)$ \\
\hline \multicolumn{2}{|l|}{ Age group, years } \\
\hline$<45$ & $106 / 264(40.2 \%)$ \\
\hline $45-54$ & $281 / 766(36.7 \%)$ \\
\hline $55-64$ & $534 / 1287(41.5 \%)$ \\
\hline $65-74$ & $501 / 1344(37.3 \%)$ \\
\hline $75-84$ & $393 / 1101(35.7 \%)$ \\
\hline $85+$ & $201 / 703(28.6 \%)$ \\
\hline \multicolumn{2}{|l|}{ Gender } \\
\hline Male & $1382 / 3606(38.3 \%)$ \\
\hline Female & $634 / 1859(34.1 \%)$ \\
\hline \multicolumn{2}{|l|}{ Ethnicity } \\
\hline Māori & $184 / 642(28.7 \%)$ \\
\hline Pacific & 80/277 (28.9\%) \\
\hline Indian & $86 / 235(36.6 \%)$ \\
\hline Other Asian & $79 / 210(37.6 \%)$ \\
\hline European/other & $1587 / 4101(38.7 \%)$ \\
\hline \multicolumn{2}{|l|}{ NZDep13 } \\
\hline $\begin{array}{l}\text { 1-2 (least } \\
\text { deprived) }\end{array}$ & $327 / 848(38.6 \%)$ \\
\hline $3-4$ & $375 / 955(39.3 \%)$ \\
\hline $5-6$ & $385 / 967(39.8 \%)$ \\
\hline $7-8$ & $452 / 1155(39.1 \%)$ \\
\hline $\begin{array}{l}\text { 9-10 (most } \\
\text { deprived) }\end{array}$ & $439 / 1261(34.8 \%)$ \\
\hline Missing & $38 / 279(13.6 \%)$ \\
\hline \multicolumn{2}{|l|}{ Hospital status } \\
\hline \multicolumn{2}{|c|}{ Primary PCI centre $1408 / 3636(38.7 \%)$} \\
\hline $\begin{array}{l}\text { Non-primary } \mathrm{PCl} \\
\text { centre }\end{array}$ & $551 / 1609(34.2 \%)$ \\
\hline $\begin{array}{l}\text { Rural hospital } \\
\text { without cath lab }\end{array}$ & $57 / 220(25.9 \%)$ \\
\hline \multicolumn{2}{|c|}{ Non-cardiac Charlson Index } \\
\hline 0 & $1551 / 3754(41.3 \%)$ \\
\hline $1-2$ & $326 / 1040(31.3 \%)$ \\
\hline $3+$ & $139 / 671(20.7 \%)$ \\
\hline \multicolumn{2}{|c|}{ Coronary intervention status } \\
\hline No cath & $270 / 1455(18.6 \%)$ \\
\hline $\begin{array}{l}\text { Cath with } \\
\text { intervention }\end{array}$ & $1574 / 3435(45.8 \%)$ \\
\hline $\begin{array}{l}\text { Cath without } \\
\text { intervention }\end{array}$ & $172 / 575(29.9 \%)$ \\
\hline
\end{tabular}

cath, catheterisation; NZDep13, New Zealand Deprivation Index 2013; PCI, percutaneous coronary intervention; STEMI, STsegment elevation myocardial infarction. had a STEMI diagnosis and $18145(24.4 \%)$ had a diagnosis of NSTEACS or other CHD (table 1). There were $57.2 \%$ diagnosed with a non-ACS condition, and $16.3 \%$ who did not require admission and for whom no further diagnostic information is available.

Outcomes for STEMI cases according to mode of transport to hospital and the initial paramedic clinical impression are shown in table 4 and figure 1 . Of the 5465 presentations with STEMI, 259 were repeat presentations which left $5206(95.3 \%)$ first STEMI presentations for outcome analysis. The characteristics of this 'first presentation' cohort are similar to those shown in table 2 for all cases (online supplemental table 3).

The unadjusted 30-day mortality rates for patients with a final diagnosis of STEMI were similar for ambulancesuspected STEMI $(9.0 \%$ (95\% CI $7.8 \%$ to $10.3 \%)$ ), suspected 'other ACS condition' $(10.9 \%$ (95\% CI $8.8 \%$ to $13.3 \%)$ ) and for self-presenters $(10.3 \%$ (95\% CI $8.8 \%$ to $12.0 \%)$ ). However, those transported by ambulance but not suspected of an ACS had the highest 30 -day mortality rate $(34.9 \%$ (95\% CI $32.2 \%$ to $37.8 \%)$, $\mathrm{p}<0.001)$. After adjusting for all demographic and clinical covariates (table 4, Model C), 30-day mortality was lower for self-presenters with STEMI than for patients with STEMI identified by ambulance paramedics (HR $0.73,95 \%$ CI 0.58 to 0.92 ). The risk for patients transported by ambulance but not initially suspected of an ACS was ameliorated but remained higher than for patients with STEMI identified by ambulance paramedics (HR 1.48, 1.22 to 1.80 ), and remained similar for ambulance-suspected 'other ACS conditions' (HR $0.85,0.65$ to 1.11 ).

\section{DISCUSSION}

St John Ambulance attends most of the call-outs in NZ and by combining its electronic dataset with the national datasets, we have a unique opportunity to assess both the accuracy of prehospital clinical impressions and their association with longer term outcomes. Only half of the patients with STEMI transported by ambulance were accurately identified and 20\% were suspected of another ACS diagnosis, with the rest not initially thought to have an ACS. Over $60 \%$ of patients with STEMI self-presented or were not identified by paramedics and so missed out on opportunities for early reperfusion by activation of the cardiac catheterisation laboratory or receipt of prehospital fibrinolysis. The unadjusted all-cause mortality was similar for ambulance-transported patients suspected of either STEMI or another ACS condition, and in those who selfpresented, but mortality was more than three times as high in those not suspected of an ACS. After adjustment for covariates, the excess mortality risk associated with not being suspected by paramedics of having a STEMI was ameliorated, but remained 1.5 times higher than for those initially identified as having a STEMI. 
Coronary artery disease

\begin{tabular}{|c|c|c|c|c|c|c|}
\hline \multirow{2}{*}{ Variables } & \multicolumn{2}{|l|}{ Model A } & \multicolumn{2}{|l|}{ Model B } & \multicolumn{2}{|l|}{ Model C } \\
\hline & HR $(95 \% \mathrm{Cl})$ & $P$ value & HR $(95 \% \mathrm{Cl})$ & $P$ value & HR $(95 \% \mathrm{Cl})$ & $P$ value \\
\hline \multicolumn{7}{|l|}{ Ambulance cardiac status } \\
\hline Suspected STEMI (ref) & 1 & - & 1 & - & 1 & - \\
\hline Other suspected ACS & 1.22 (0.94 to 1.59$)$ & 0.14 & 1.11 (0.85 to 1.44$)$ & 0.45 & 0.85 (0.65 to 1.11$)$ & 0.22 \\
\hline ACS not suspected & 4.41 (3.69 to 5.27 ) & $<0.01$ & 3.29 (2.74 to 3.95$)$ & $<0.01$ & $1.48(1.22$ to 1.80$)$ & $<0.01$ \\
\hline Not attended by ambulance & 1.14 (0.92 to 1.42$)$ & 0.24 & 1.23 (0.99 to 1.54$)$ & 0.06 & 0.73 (0.58 to 0.92$)$ & 0.01 \\
\hline \multicolumn{7}{|l|}{ Demographics } \\
\hline Age, per year & - & - & 1.045 (1.039 to 1.051$)$ & $<0.01$ & 1.015 (1.008 to 1.022$)$ & $<0.01$ \\
\hline Sex & - & - & & & & \\
\hline Male & & & 0.99 (0.86 to 1.15$)$ & 0.94 & 1.14 (0.98 to 1.32$)$ & 0.09 \\
\hline Female (ref) & & & 1 & - & 1 & - \\
\hline Ethnicity & - & - & - & - & & \\
\hline Māori & & & & & 1.09 (0.84 to 1.41$)$ & 0.53 \\
\hline Pacific & & & & & 1.27 (0.92 to 1.76$)$ & 0.15 \\
\hline Indian & & & & & 0.94 (0.58 to 1.53$)$ & 0.81 \\
\hline Asian & & & & & 0.78 (0.48 to 1.27$)$ & 0.31 \\
\hline NZ Euro/other (ref) & & & & & 1 & - \\
\hline NZDep13 & - & - & - & - & & \\
\hline 1-2 (ref) & & & & & 1 & - \\
\hline $3-4$ & & & & & 1.09 (0.84 to 1.41$)$ & 0.51 \\
\hline $5-6$ & & & & & 1.24 (0.97 to 1.60$)$ & 0.09 \\
\hline $7-8$ & & & & & $1.27(1.00$ to 1.62$)$ & 0.05 \\
\hline $9-10$ & & & & & $1.33(1.04$ to 1.70$)$ & 0.02 \\
\hline $\begin{array}{l}\text { Modified Charlson Comorbidity Index } \\
0 \text { (ref) }\end{array}$ & - & - & - & - & & \\
\hline $1-2$ & & & & & 1 & - \\
\hline \multirow[t]{2}{*}{$\geq 3$} & & & & & 1.01 (0.84 to 1.21$)$ & 0.91 \\
\hline & & & & & 1.24 (1.03 to 1.49$)$ & 0.02 \\
\hline Coronary intervention status & - & - & - & - & & \\
\hline No angiography (ref) & & & & & 1 & - \\
\hline Angiography without intervention & & & & & 0.25 (0.19 to 0.34$)$ & $<0.01$ \\
\hline Angiography with intervention & & & & & 0.12 (0.10 to 0.16$)$ & $<0.01$ \\
\hline Hospital status & - & - & - & - & & \\
\hline Routine 24/7 PCl primary PCl & & & & & 1.13 (0.96 to 1.33$)$ & 0.13 \\
\hline Base hospital w/0 24/7 PCl (ref) & & & & & 1 & - \\
\hline Rural hospital & & & & & 1.04 (0.76 to 1.44$)$ & 0.8 \\
\hline
\end{tabular}

Model A: unadjusted.

Model B: adjusted by age and sex.

Model C: adjusted for age, sex, ethnicity, NZDep quintile, non-cardiac comorbidity $(0,1-2,>2)$, coronary intervention status (no angiography, angiography without intervention, angiography with intervention), hospital status (primary $\mathrm{PCl}$, non-primary $\mathrm{PCl}$ with catheterisation lab, hospital without catheterisation lab).

ACS, acute coronary syndrome; NZDep13, New Zealand Deprivation Index 2013; PCI, percutaneous coronary intervention; STEMI, STsegment elevation myocardial infarction.

\section{Demographic differences}

Māori (the indigenous population of NZ) and Pacific patients with a final hospital STEMI diagnosis were less likely to have been suspected of having a STEMI by paramedics compared with other ethnic groups. They were also more likely to have self-presented to hospital rather than called an ambulance. As a result, Māori and Pacific groups are less likely to receive timely STEMI 


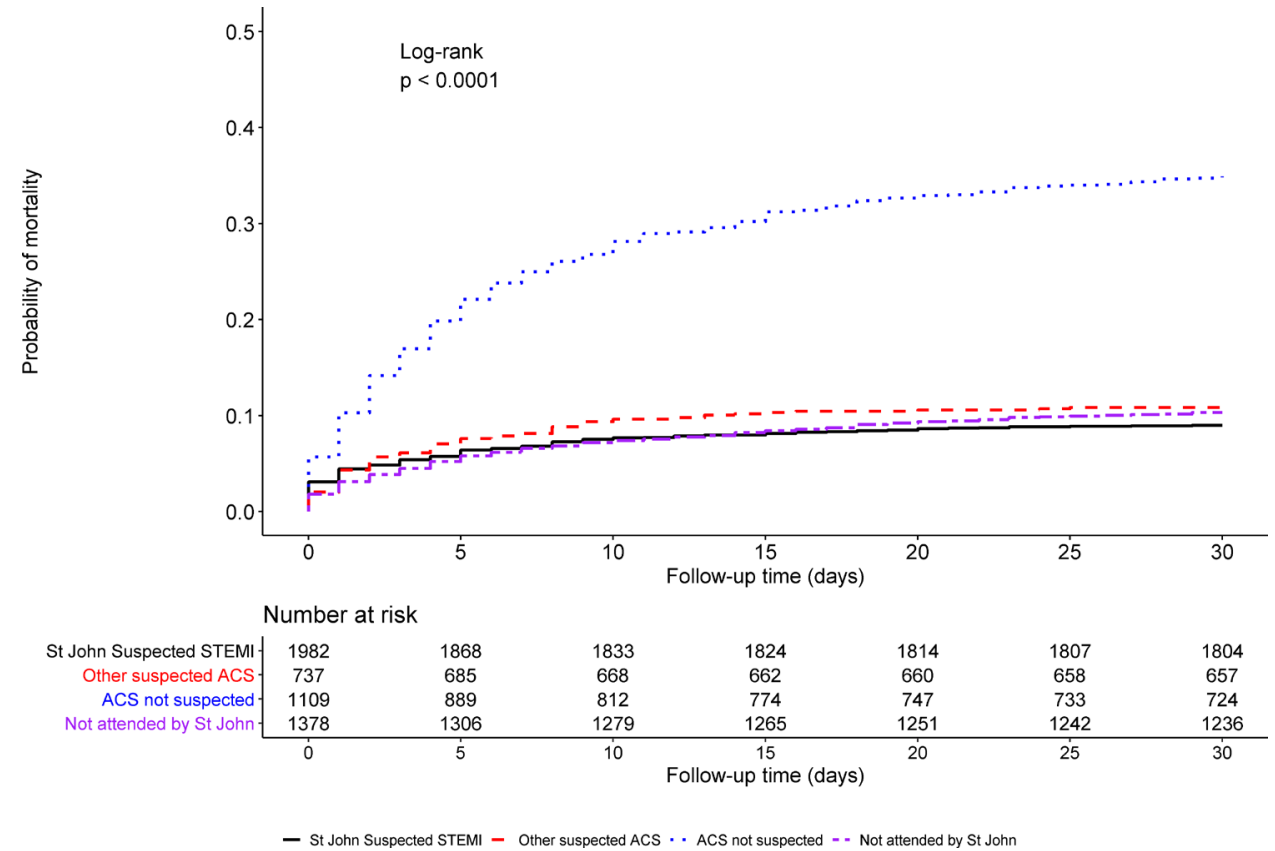

Figure 1 Unadjusted all-cause mortality for patients with STEMI* according to method of patient transport and initial ambulance paramedic clinical impression. *For patients with more than one STEMI presentation, only the first presentation was used in the outcomes analysis. ACS, acute coronary syndrome; STEMI. ST-segment elevation myocardial infarction.

therapy which is critical for improving clinical outcomes. This may contribute to their documented poorer outcomes post-MI. ${ }^{13}$

Patients with STEMI admitted to routine primary PCI-capable hospitals were more likely identified with STEMI by paramedics, compared with non-routine PCI-capable or rural hospitals $(53 \%, 46 \%$ and $36 \%$, respectively). While this may be due to factors such as paramedic training and appropriate utilisation of ECGs at the scene, the findings are also biased by patients with very clear STEMI in rural hospital catchments being transported directly to primary PCI-capable hospitals, leaving more equivocal cases going to rural hospitals. Prior analyses have not found differences in all-cause mortality based on the type of initial receiving hospital which may be due to appropriate identification and bypass of high-risk patients directly to primary PCI centres. ${ }^{14}$

\section{Ambulance versus self-transport}

Seventy-three per cent of the patients with STEMI were transported to hospital by ambulance. This compares favourably with international data. An observational analysis of 37634 patients with STEMI treated at over 300 US hospitals found that emergency medical services were used for $59 \%-68 \%$ of patients. ${ }^{15-17}$ Nevertheless, in the current study, 27\% of the STEMI cases were not attended by St John, a sizeable group without prehospital access to acute care and defibrillators. These patients will have longer time to reperfusion treatment as previously reported in the ANZACS-QI registry and from the REACT trial. ${ }^{18} 19$
Paramedic diagnostic accuracy and implications

Prehospital clinical impression of STEMI was confirmed in 57\% of cases. Prior studies have reported higher diagnostic accuracies for STEMI. One study of 354 patients had suspected ACS assessed by their mobile emergency care unit (ambulance with two emergency medical staff) at $87.5 \%$ of preliminary STEMI diagnoses confirmed. ${ }^{20}$ Another Danish study similarly reported accuracy of $89 \% .{ }^{21}$ A third study assessed 103 paramedics' responses to five different settings of chest pain and ECGs, finding 93\% sensitivity and $85 \%$ specificity for paramedic diagnosis of STEMI. ${ }^{22}$ In contrast, another cohort study using paramedics' surveys reported a lower sensitivity $75 \%$ and specificity $53 \%$ for STEMI detection, including $96 \%$ correct for inferior STEMI but only $51 \%$ correct for lateral STEMI, and no correlation between paramedic training, experience and accuracy. ${ }^{22}$

\section{Outcomes}

Patients with STEMI who were transferred by ambulance but not initially suspected of an ACS had over three times the mortality risk of those initially identified by paramedics as STEMI. The adverse outcomes are in part due to covariates including age and comorbidities; and after adjustment, the excess hazard was markedly reduced to just 1.5 times excess risk. Some of this residual excess risk may be related to unmeasured and therefore unadjusted comorbidity. In particular, the national datasets do not have information about clinical acuity at the time of presentation, and it is possible that some patients with STEMI without a clear STEMI diagnosis may be more unwell. We are unable to determine, in this observational 
study, whether some of the remaining excess risk is due to the delay in appropriate monitoring and treatment for these patients. It is also possible that patients who call an ambulance may be sicker than those who self-present. This is supported by the finding that after adjustment, self-presenters had better outcomes than ambulancetransferred patients.

\section{Clinical implications}

Increasing both the proportion transferred by ambulance and improving identification of at-risk patients by paramedics may improve prehospital STEMI identification. This may facilitate earlier reperfusion therapy whether primary PCI or fibrinolysis. Consistent and increased utilisation of prehospital ECGs may help identify at-risk patients earlier. Ongoing training of paramedics on ECG interpretation and recognition of ACS-related symptoms is needed. The relatively low positive predictive value of $57 \%$ of the ambulance clinical impression of STEMI is of concern, and needs to be addressed as prehospital fibrinolysis programmes are rolled out, to avoid patient harm related to unnecessary fibrinolysis. Strategies to increase public awareness of ACS could include media campaigns, advanced cardiac life support community courses and school programmes, with goals of shortening the time to calling an ambulance and enhancing ambulance utilisation. The data linkage methodology used for this study will allow routine audit and ongoing quality improvement initiatives.

\section{Limitations}

Patients who died prehospital were not included in this study as final diagnosis data depended on hospital admission. Some St John records in the initial dataset did not have NHIs so could not be included in this study. Records with missing NHIs were predominantly for patients not transported to hospital (personal communication, Bridget Dicker). Some patients may have developed STEMI in hospital therefore could not have been identified by St John. However, using additional data available for nearly $80 \%$ of the patients with STEMI, we could estimate how many would have had a delayed diagnosis due to the ECG findings developing after admission. Exclusion of those patients would have increased the sensitivity of the St John clinical impression from $51 \%$ to 53\%. Although the available data did not record the exact proportion of patients who did not receive a prehospital ECG but prior audit from St John Ambulance has shown 99\% of suspected cardiac cases had a 12-lead ECG (personal communication, Bridget Dicker). Our study results were based on real-world national experience of suspected STEMI management in NZ, with our own unique patient population and healthcare systems, therefore findings are not necessarily generalisable to other countries and healthcare systems.

\section{CONCLUSION}

This large national data linkage study found over 6 out of 10 patients with STEMI potentially missed out on early reperfusion due to self-presentation to hospital or not being identified by paramedics as having a STEMI. Patients with STEMI transported by ambulance but not initially suspected of having an ACS had worse prognosis, in part explained by differences in baseline demographic and clinical factors.

\section{Author affiliations}

${ }^{1}$ Department of Cardiology, Middlemore Hospital, Auckland, New Zealand ${ }^{2}$ Greenlane Cardiovascular Services, Auckland City Hospital, Auckland, New Zealand ${ }^{3}$ Paramedicine Research Unit, Paramedicine Department, School of Clinical Sciences, Auckland University of Technology, Auckland, New Zealand

${ }^{4}$ Clinical Audit and Research, St John New Zealand, Auckland, New Zealand ${ }^{5}$ Section of Epidemiology and Biostatistics, School of Population Health, University of Auckland, Auckland, New Zealand

${ }^{6}$ School of Medicine, University of Auckland, Auckland, New Zealand

Acknowledgements We would like to thank the Clinical Audit and Research team at St John and the Middlemore Cardiac Trust for their support of this study. ANZACS-QI programme implementation, coordination and analysis: the ANZACS-QI software was developed and supported by Enigma Solutions. Programme implementation is coordinated by the National Institute for Health Innovation (NIHI) at the University of Auckland. The ANZACS-QI programme is funded by the New Zealand Ministry of Health. We thank the National Health Board Analytic Services enabling use of the national datasets. We also thank the VIEW team at the School of Population Health, University of Auckland, for the curation and linkage of the national data. ANZACS-QI Governance Group: Andrew Kerr (chair), Dean Boddington, Gary Sutcliffe, Gerry Devlin, Harvey White, John Edmond, Jonathon Tisch, Kim Marshall, Mayanna Lund, Michael Williams (deputy chair), Nick Fisher, Seif El Jack and Sue Riddle. ANZACS-QI Project management: Kristin Sutherland (project manager), Charmaine Flynn (northern coordinator) and Maxine Rhodes (southern coordinator). Data analysis: Mildred Lee. Data management: Billy Wu (SOPH), Michelle Jenkins (NIHI) and John Faatui (NIHI). We acknowledge all the New Zealand cardiologists, physicians, nursing staff, radiographers and patients who have supported and contributed to ANZACS-QI.

Contributors Study design—BY-WL and AK. Data collection-all co-authors. Data analysis-BY-WL, MAWL and AK. Data interpretation-all co-authors. Manuscript writing - BY-WL and AK. Critical review of the manuscript and final approval of the manuscript-all c0-authors. Guarantor- AK.

Funding This work was supported by the New Zealand Health Research Council, Wellington (grant number 11/800 to AK). KP is supported by a New Zealand Heart Foundation Hynds Senior Fellowship. BY-WL and MAWL are supported by the Middlemore Hospital Cardiology Trust.

Disclaimer Researchers are independent from funders, and the funders had no role in the study design, collection, analysis or interpretation of data.

Competing interests None declared.

Patient consent for publication Not required.

Ethics approval ANZACS-QI is part of the Vascular Informatics Using Epidemiology and the Web (VIEW) programme. The VIEW programme was approved by the Northern Region Ethics Committee $Y$ in 2003 (AKY/03/12/314), with subsequent amendments to include the ANZACS-QI registries, and with annual approval by the National Multi-region Ethics Committee since 2007 (MEC07/19/EXP). The St John Ambulance service locality review approved the use of St John data in this study. All data were anonymised in the analyses so individual patient consent was not required. The corresponding author affirms that the manuscript is an honest, accurate and transparent account of the study being reported, that no important aspects of the study have been omitted.

Provenance and peer review Not commissioned; externally peer reviewed.

Data availability statement Data are available upon reasonable request. The data underlying this article cannot be shared publicly because they are made available to the investigators by the Ministry of Health under a Multi-region Ethics Committee 
approval. The data will be shared on reasonable request to the corresponding author and approval by the VIEW Governance Group.

Open access This is an open access article distributed in accordance with the Creative Commons Attribution Non Commercial (CC BY-NC 4.0) license, which permits others to distribute, remix, adapt, build upon this work non-commercially, and license their derivative works on different terms, provided the original work is properly cited, appropriate credit is given, any changes made indicated, and the use is non-commercial. See: http://creativecommons.org/licenses/by-nc/4.0/.

\section{ORCID iDs}

Becky Yi-Wen Liao http://orcid.org/0000-0001-9910-013X

Bridget Dicker http://orcid.org/0000-0002-8151-7356

Ralph Stewart http://orcid.org/0000-0002-6167-1225

Katrina Poppe http://orcid.org/0000-0002-4418-4476

\section{REFERENCES}

1 Roffi M, Patrono C, Collet J-P, et al. 2015 ESC guidelines for the management of acute coronary syndromes in patients presenting without persistent ST-segment elevation: Task force for the management of acute coronary syndromes in patients presenting without persistent ST-segment elevation of the European Society of cardiology (ESC). Eur Heart $J$ 2016;37:267-315.

2 Ibanez B, James S, Agewall S, et al. 2017 ESC guidelines for the management of acute myocardial infarction in patients presenting with ST-segment elevation: the task force for the management of acute myocardial infarction in patients presenting with ST-segment elevation of the European Society of cardiology (ESC). Eur Heart $J$ 2018;39:119-77.

3 Levine GN, Bates ER, Blankenship JC. ACCF/AHA/SCAI guideline for percutaneous coronary intervention: a report of the American College of cardiology Foundation/American heart association Task force on practice guidelines and the Society for cardiovascular angiography and interventions. Circulation 2011;2011:e574-651.

4 Amsterdam EA, Wenger NK, Brindis RG. ACC/AHA Task force members; Society for cardiovascular angiography and interventions and the Society of thoracic surgeons. 2014 AHA/ACC guideline for the management of patients with non-ST-elevation acute coronary syndromes: Executive summary: a report of the American College of Cardiology/American heart association Task force on practice guidelines. Circulation 2014:130:2354-94.

5 Johnston S, Brightwell R, Ziman M. Paramedics and pre-hospital management of acute myocardial infarction: diagnosis and reperfusion. Emerg Med J 2006;23:331-4.

6 Keeley EC, Hillis LD. Primary PCl for myocardial infarction with STsegment elevation. N Engl J Med 2007;356:47-54.

7 Keeley EC, Boura JA, Grines CL. Primary angioplasty versus intravenous thrombolytic therapy for acute myocardial infarction: a quantitative review of 23 randomised trials. Lancet 2003;361:13-20.

8 McNamara RL, Wang Y, Herrin J, et al. Effect of door-to-balloon time on mortality in patients with ST-segment elevation myocardial infarction. J Am Coll Cardiol 2006;47:2180-6.
9 Kerr AJ, Lee M, Jiang Y, et al. High level of capture of coronary intervention and associated acute coronary syndromes in the all New Zealand acute coronary syndrome quality improvement cardiac registry and excellent agreement with national administrative datasets (ANZACS-QI 25). N Z Med J 2019;132:19-29.

10 Kerr A, Williams MJ, White $\mathrm{H}$, et al. The all New Zealand acute coronary syndrome quality improvement programme: implementation, methodology and cohorts (ANZACS-QI 9). N Z Med J 2016;129:23-36

11 Atkinson J, Salmond C, Crampton P. NZDep2013 index of deprivation. Wellington: Department of Public Health, University of Otago, 2014.

12 HISO 10001:2017 ethnicity data protocols, 2017. Available: https:// www.health.govt.nz/publication/hiso-100012017-ethnicity-dataprotocols

13 Mazengarb J, Grey C, Lee M, et al. Inequity in one-year mortality after first myocardial infarction in Māori and Pacific patients: how much is associated with differences in modifiable clinical risk factors? (ANZACS-QI 49). N Z Med J 2020;133:40-54.

14 Lee S, Miller R, Lee M, et al. Outcomes after ST-elevation myocardia infarction presentation to hospitals with or without a routine primary percutaneous coronary intervention service (ANZACS-QI 46). N Z Med J 2020;133:64-81.

15 Kaul P, Welsh RC, Liu W, et al. Temporal and provincial variation in ambulance use among patients who present to acute care hospitals with ST-elevation myocardial infarction. Can J Cardiol 2016;32:949-55

16 So DYF, Ha ACT, Turek MA, et al. Comparison of mortality patterns in patients with ST-elevation myocardial infarction arriving by emergency medical services versus self-transport (from the prospective Ottawa Hospital STEMI registry). Am J Cardiol 2006;97:458-61.

17 Kerr A, Lee M, Grey C, et al. Acute reperfusion for ST-elevation myocardial infarction in New Zealand (2015-2017): patient and system delay (ANZACS-QI 29). N Z Med J 2019;132:41-59.

18 Shiomi H, Morimoto T, Kitaguchi S, et al. The ReACT Trial: Randomized Evaluation of Routine Follow-up Coronary Angiography After Percutaneous Coronary Intervention Trial. JACC Cardiovasc Interv 2017;10:109-17.

19 Hansen LH, Mikkelsen S. Ischaemic heart disease: accuracy of the prehospital diagnosis-a retrospective study. Emerg Med Int 2013;2013:754269

20 Andersen MS, Nielsen TT, Christensen EF. A study of police operated dispatch to acute coronary syndrome cases arising from 112 emergency calls in Aarhus County, Denmark. Emerg Med J 2006;23:705-6.

21 Trivedi K, Schuur JD, Cone DC. Can paramedics read STsegment elevation myocardial infarction on prehospital 12-lead electrocardiograms? Prehosp Emerg Care 2009;13:207-14.

22 Mencl F, Wilber S, Frey J, et al. Paramedic ability to recognize ST-segment elevation myocardial infarction on prehospital electrocardiograms. Prehosp Emerg Care 2013;17:203-10. 